\title{
The Effect of Fiber Geometry and Interfacial Properties on the Elastic Properties of Cementitious Nanocomposite Material
}

\author{
Ala G. Abu Taqa, ${ }^{1}$ Rashid K. Abu Al-Rub, ${ }^{2}$ Ahmed Senouci, ${ }^{3}$ \\ Nasser Al-Nuaimi, ${ }^{1}$ and Khaldoon A. Bani-Hani ${ }^{4}$ \\ ${ }^{1}$ Department of Civil and Architectural Engineering, Qatar University, P.O. Box 2713, Doha, Qatar \\ ${ }^{2}$ Institute Center for Energy, Mechanical and Materials Engineering Department, Masdar Institute of Science and Technology, \\ P.O. Box 54224, Abu Dhabi, UAE \\ ${ }^{3}$ Department of Construction Management, University of Houston, Houston, TX 77204-4020, USA \\ ${ }^{4}$ Department of Civil Engineering, Jordan University of Science and Technology, P.O. Box 3030, Irbid 22110, Jordan
}

Correspondence should be addressed to Ala G. Abu Taqa; aal104819@qu.edu.qa

Received 27 January 2015; Accepted 21 March 2015

Academic Editor: Hassan Karimi-Maleh

Copyright ( 2015 Ala G. Abu Taqa et al. This is an open access article distributed under the Creative Commons Attribution License, which permits unrestricted use, distribution, and reproduction in any medium, provided the original work is properly cited.

\begin{abstract}
This paper investigates the elastic (Young's) modulus of carbon Nanotube- (CNT-) reinforced cement paste using 3D and axisymmetric models using Abaqus software. The behavior of the CNT and the cement matrix was assumed to be fully elastic while the cohesive surface framework was used to model the interface. To investigate the effect of fiber waviness on the value of the elastic modulus, 3D models were developed assuming different distributions of fibers. The results obtained using the 3D model were compared to those obtained using the simplified three-phase axisymmetric model which consists of one single CNT aligned in the center of composite unit cell, an interface, and cement matrix. A parametric study was then carried out using the axisymmetric model to study the role of the interface in the composite elastic modulus without accounting for the presence of the interfacial transition zone (ITZ or interphase). The results showed that the CNTs waviness significantly reduced their reinforcing capability in the cement paste. On the other hand, the results obtained using the axisymmetric model were found to be in good agreement with those obtained using the 3D model. Moreover, the results of the parametric study showed that the interface properties significantly affect the composite elastic modulus and alter its behavior.
\end{abstract}

\section{Introduction}

Since their discovery in 1990s [1], CNTs have shown an excellent potential to be used in composites because of their special physical properties resulting from the symmetry of their structure. CNTs occur as single-walled carbon nanotubes (SWNTs) and multiwalled carbon nanotubes (MWNTs). SWNTs are composed of a single graphite sheet rolled into a long hollow cylinder, whereas MWNTs are nested arrays of SWNTs. The average diameter of an individual SWNT is on the order of $1 \mathrm{~nm}$ whereas the average diameter of an individual MWNT is on the order of $10 \mathrm{~nm}$. Both SWNTs and MWNTs have aspect ratios of approximately 1000. CNTs elastic modulus is about $1 \mathrm{TPa}$, which is slightly lower than that of diamond $(1.2 \mathrm{TPa})$ and could be considered as one of the highest reported elastic moduli among existing materials. Moreover, their strength is about 1 to 2 orders of magnitude higher than that of high strength steel alloy, their thermal conductivity is almost two times that of diamond [2], and their current carrying capacity is 1000 times higher than copper [3].

The utilization of CNTs has attracted the researchers in all scientific disciplines. For example, CNTs were used as sensors in human and medical science [4-8]. They were also utilized in food safety discipline for the determination of some chemicals in food samples which prevent the foodborne illnesses [9]. Moreover, CNTs have the capability of producing new class of composites with upgraded qualities because of their high fracture strain capacity, low density, and elastic behavior under large strains. 
Theoretical studies claimed significant improvements in CNT-reinforced composites. However, experimental results showed considerably lower level of improvement in the properties of nanoreinforced composites. The marginal improvement witnessed experimentally might be due to the poor dispersion of carbon nanotubes, weak interfacial bonding between matrix and CNTs, agglomeration of the CNTs, and/or defects generated during the processing of carbon nanotubes. Moreover, CNTs waviness is one of the main features that limits their effectiveness. CNTs have high aspect ratio and low bending stiffness in the direction perpendicular to their axis. This allows them to bend and twist easily within the matrix and results in composite with mechanical properties significantly lower than composite with straight fibers.

Significant research work has been done to account for the reduction in the composite stiffness due to the waviness of fibers. Hsiao and Daniel [10] proposed an analytical model to determine the effective elastic modulus of laminated composites with wavy fibers along the laminates using a representative volume element (RVE). The study was limited to the case of laminated composites with fibers aligned in the axial direction. The authors reported that Young's modulus of the specimen is significantly decreased due to the waviness of the fibers while its Poisson's ratio is increased. Fisher et al. [11] conducted a more comprehensive study on the influence of waviness on nanotube-reinforced polymers. They tried to extend the conventional micromechanical theory in analyzing the composites using finite element method. An effective reinforcing modulus (ERM) parameter was used to quantify the stiffness reduction of nanotubes due to randomly oriented sinusoidal waviness. In their study, Bradshaw et al. [12] replaced curved inclusions by straight ones with reduced stiffness $\left(E_{\mathrm{ERM}} \leq E_{N T}\right)$. The Mori-Tanaka method was used to model the composite with randomly oriented straight inclusions. Fisher et al. [11] used a more robust approach that models the exact $3 \mathrm{D}$ shape of the nanotubes to determine the elastic modulus of nanotube-reinforced composites. This method is more complicated and computationally more expensive. Both studies showed an important reduction in Young's modulus when the waviness increased. However, both approaches overestimated the elastic modulus results compared to those obtained experimentally. This is due to the fact that these models incorporated simplifications that resulted in the stiffening of the composite. Shi et al. [13] studied the same issue considering a helical shape for the nanotubes. They also investigated the effect of agglomeration on the elastic properties of CNT-reinforced composites. The results showed that both waviness and agglomeration significantly reduced the stiffening effect of the CNTs. Shao et al. [14] also studied the effect of waviness using same approach used by Bradshaw et al. [12] and Fisher et al. [11] considering three replacements for the curved inclusions, namely, arc of a circle, sinusoid, and parabola. They also tried to incorporate the effect of debonding or weak bonding between the nanotubes and the matrix, using simple analytical models. Spanos and Esteva [15] proposed a new approach for computing the effect of nanotube waviness, which is more computationally efficient than the previously mentioned ones. The study used the embedment method which entrenches the fiber within the matrix using a set of constraints as explained by Ranjbaran [16]. In this method, the fiber geometry and distribution are not considered in the mesh generation procedure to reduce the amount of computational effort required to analyze nanotube-reinforced composites. However, in order to obtain fairly accurate results the mesh should be fine enough.

The above studies addressed mostly nanoreinforced polymers. The effect of CNTs waviness on the mechanical properties of cement paste is still not well understood. The results obtained from theoretical studies showed significant improvements in CNT-reinforced cement paste. However, these results did not agree with those obtained experimentally. The experimental results showed only a marginal improvement in the mechanical properties of the CNTreinforced cement paste [17]. As mentioned before, this might be due to the simplifying assumptions used in the theoretical studies.

On the other hand, modeling the stiffness and strength of the nanocomposite cement paste needs to consider the mechanical properties of the region surrounding the CNT which significantly influence the bulk composite properties including the elastic modulus. This region is characterized by two zones, namely, interfacial transition (ITZ or interphase) and interface. The interface, which is defined as a cohesive surface with zero thickness, lies between the CNT and the interphase. The interphase, which has a finite thickness, has more distinct mechanical properties than those of the $\mathrm{CNT} /$ cement interface and the cement matrix.

The knowledge about the interfacial behavior between the CNTs and the cement matrix is very limited. While many have been reported in CNT/polymer composites, little experimental work has been conducted to investigate the interfacial properties in cementitious nanocomposites, which mainly control the load transfer mechanism between the CNT and the cement matrix (if the friction after debonding is ignored). For example, Chandra [18] computed the fundamental cohesive zone parameters such as traction and displacement for the CNT/polymer composites using molecular dynamics. He showed that the interface strength value ranges from $500 \mathrm{MPa}$ to $5 \mathrm{GPa}$ depending upon the density of the chemical bonds per unit length of the CNT. However, Naaman et al. [19] evaluated the interfacial shear strength between steel fiber and cement-based matrices in steel fiber reinforced concrete (SFRC) from experimental pullout curves, and the obtained values were in the range of $1.4 \mathrm{MPa}$ to $9.6 \mathrm{MPa}$. Chan and Andrawes [21] reported an effective shear strength value of $6.5 \mathrm{MPa}$ between CNTs and cement by calibrating the numerical models using empirical results from three-point bending tests. Chan and Andrawes [20] also conducted a parametric study by varying the interfacial shear strength values from $5 \mathrm{MPa}$ to $20 \mathrm{MPa}$.

From economical (time and costs) point of view, performing straightforward macro- and microexperimental measurements on a number of material samples of various particle sizes, types, shapes, volume fractions, and dispersions/distributions under different loading conditions is a hardly feasible task. However, at the present time, it is still 
not possible to perform atomistic or molecular dynamics simulations (MD) on realistic time scale and structures. Therefore, in this study, a multiscale computational framework is developed to study the effect of fiber waviness and the interfacial properties on the overall elastic response of the nanocomposite cement.

This paper has two main objectives. The first one is investigating the effect of CNTs waviness on the elastic properties of nanocomposite cement using 3D RVE maker model which randomly generates nanotubes in a $3 \mathrm{D}$ matrix. The approach used in creating the fibers in 3D models is similar to the one proposed for the study of electrical conductivity in 3D entangled fibrous networks [22]. An axisymmetric unit cell model was created with appropriate boundary conditions to simplify the 3D models. The results of the 3D simulation were compared to those obtained using the axisymmetric configuration assuming a perfect bond between the CNT and the matrix and ignoring the presence of the interphase or ITZ. The second objective consists of conducting a parametric study to explore the effect of the interfacial properties (namely, initial stiffness, cohesive shear strength, and maximum allowable slip or cohesive energy) on the elastic response of the nanocomposite cement using the axisymmetric unit cell model for a single aligned CNT embedded in the cement matrix. In this paper, the behavior of the CNT and cement matrix is assumed to be linearly elastic. On the other hand, the interface is modeled using a cohesive surface model neglecting the interfacial friction after debonding. Assuming an elastic behavior for the CNTs is fairly reasonable due to their high axial stiffness while it sounds questionable for the cement paste. However, this assumption is acceptable herein because the focus of this study is to investigate the overall composite elastic modulus only rather than its strength and ductility. This work can be extended to incorporate the plasticity and damage behavior of the cement paste.

\section{Modeling and Assumptions}

3D and axisymmetric models were developed herein to investigate the enhancement of the elastic modulus of the CNTreinforced cement paste. The behavior of the cement paste and the CNTs is considered to be fully elastic and the CNTs are modeled as beams. Therefore, the hollow nature of the tubes is not considered. Moreover, the interlayer movement of the multiwalled nanotubes (MWNTs) is neglected.

2.1. 3D Models. 3D models were constructed using an RVE maker developed by Tehrani and Abu Al-Rub [23] which randomly generates $\mathrm{CNT}$ distribution within a representative volume. The dimensions of the RVE were chosen equal to $1.5 \times 1.5 \times 1.5 \mu^{3}$. The planes located at $X=0, Y=0$, and $Z=0$ are restrained in the $X-, Y$-, and $Z$-directions, respectively. A displacement of $-0.15 \mu \mathrm{m}$ is applied at the top surface (face), $Z=1.5 \mu \mathrm{m}$. The mechanical properties of the matrix and the fiber are shown in Table 1.

Two CNT volume fractions (VF) of $0.1 \%$ and $0.5 \%$ were used. The ranges of length and diameter of randomly dispersed CNTs were $1-1.2 \mu \mathrm{m}$ and $8-12 \mathrm{~nm}$, respectively.
TABLE 1: Mechanical properties of the matrix and fibers.

\begin{tabular}{lccc}
\hline & \multicolumn{2}{c}{ Elastic modulus $(E)$} & \multirow{2}{*}{ Poisson's ratio $(v)$} \\
& $\mathrm{GPa}$ & $\mathrm{N} /(\mathrm{nm})^{2}$ & \\
\hline Cement matrix & 30 & $3 \times 10^{-8}$ & 0.20 \\
CNT & 1000 & $1 \times 10^{-6}$ & 0.35 \\
\hline
\end{tabular}

Three cases were considered for each volume fraction. The fibers were straight and dispersed randomly in $Z$ direction in the first case. On the other hand, the fibers were considered straight and curved in the second and third cases, respectively, and they were randomly dispersed in arbitrary directions within the matrix. The curved fibers were constructed using the same concept that was proposed in Dalmas et al. [22] in order to avoid any intersections between them. Periodic boundary conditions have not yet been implemented in the RVE maker. Therefore, none of the fibers intersected the boundaries of the RVE. The generated models and their boundary conditions for volume fraction $\mathrm{VF}=0.1 \%$ are shown in Figure 1 .

2.2. Axisymmetric Model. The 3D representative volume element (RVE) of the cement nanocomposite with straight CNTs distributed randomly in the direction parallel to the applied load (Figure 1(a)) can be simplified using an equivalent axisymmetric configuration. The RVE is first divided into unit cells having hexagonal cross section and containing a single carbon nanotube at its center. Then, the hexagonal cross section could be replaced by an equivalent circular cross section to simulate an axisymmetric unit cell. In this study, the axisymmetric model consisted of three phases, namely, CNT, interface, and the cement matrix. The interfacial transition zone (ITZ or interphase) was ignored as shown in Figure 2. The commercial finite element software Abaqus was used for the analysis. A 4-node bilinear axisymmetric quadrilateral element with reduced integration (CAX4R) was used for the CNT and cement matrix while the cohesive surface framework was used to model the interface. To compare the results of the $3 \mathrm{D}$ and axisymmetric models, the CNT and cement matrix were assumed to be perfectly bonded at the interface to have a complete load transfer from the matrix yielding ideal composite material properties. This represents the upper bound for the interfacial strength which is infinity.

2.2.1. Geometry. The unit cell length was taken equal to $2 L_{C}=$ $1.5 \mu \mathrm{m}$ (equivalent to the length of the 3D RVE). The length and diameter of the CNT were taken equal to $2 L_{\mathrm{CNT}}=1.1 \mu \mathrm{m}$ and $2 R_{\mathrm{CNT}}=10 \mathrm{~nm}$. The unit cell radius, $R_{C}$, is determined for each CNT volume fraction using the following equation:

$$
f_{\mathrm{CNT}}=\frac{R_{\mathrm{CNT}}^{2} L_{\mathrm{CNT}}}{R_{\mathrm{C}}^{2} L_{\mathrm{C}}},
$$

where $f_{\mathrm{CNT}}$ is the CNT volume fraction.

However, to investigate the effect of the CNT aspect ratio in the parametric study, the unit cell radius was fixed as per the CNT volume fraction selected for the study while the 


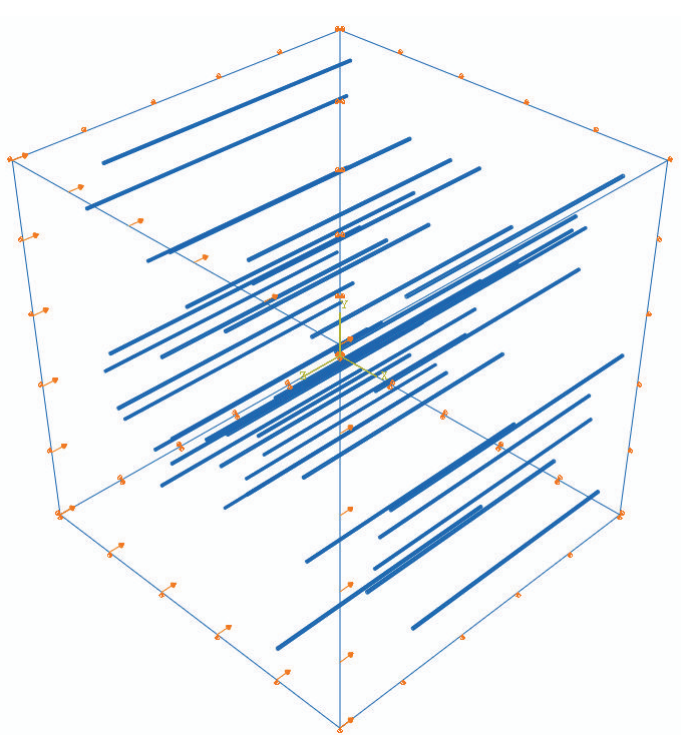

(a)

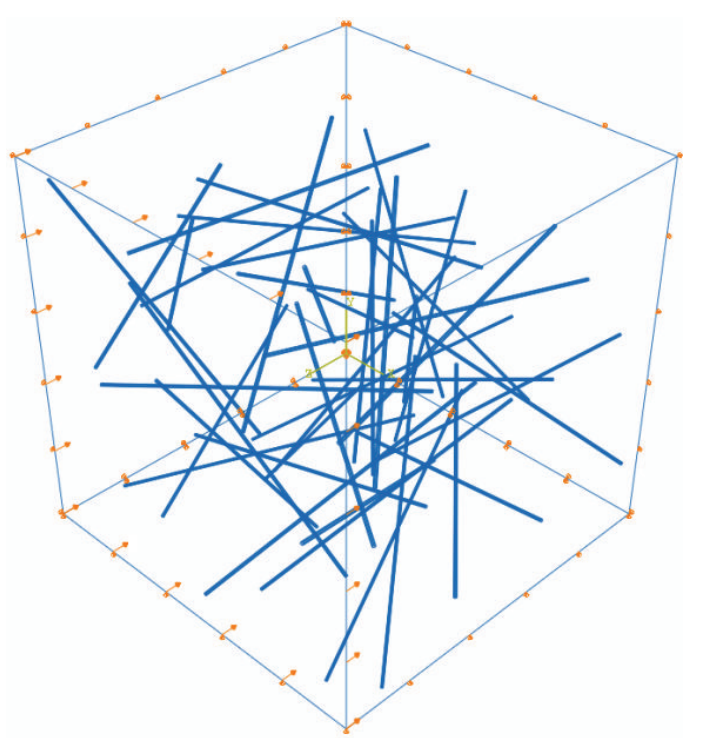

(b)

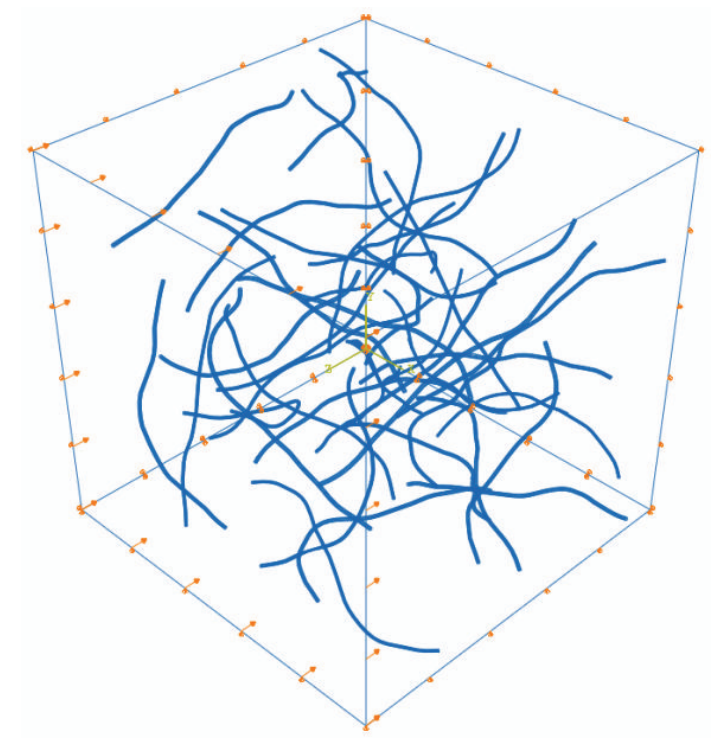

(c)

FIGURE 1: Geometry and boundary conditions for 3D models: (a) Case 1, (b) Case 2, and (c) Case 3.

length of the CNT was changed to generate different aspect ratios.

\subsubsection{Boundary Conditions}

(a) At $z=L_{C}$. A tension displacement of $0.075 \mu \mathrm{m}$ was applied at $z=L_{C}$ when working on the first objective. On the other hand, the computations were carried out up to an axial strain of 0.0005 (equivalent to a tension displacement of $0.375 \mathrm{~nm}$ ). The computation range was limited to this small strain value to ensure that the CNT was not fully debonded from the cement matrix even for smaller interfacial strength values. Hence, the results of the elastic modulus values in the parametric study can be compared. The displacement was applied as displacement rate at small time increments in order to set the time scale, achieve the convergence, and get more accurate stress-strain diagram in the elastic region.

(b) At $r=R_{C}$. The boundary condition $u_{r}\left(R_{C}, z\right)=W$ was applied, where $W$ was determined from the condition $\sum r=$ 0 , and $\sum r$ was the average stress in the radial direction. This boundary condition was enforced by running the model first without applying any pressure at the boundary $r=R_{C}$. The average radial stress was calculated at this boundary. Then, a pressure opposite and equal to the average radial stress was applied at the boundary.

2.2.3. Meshing. Different mesh sizes were investigated to check the sensitivity of the analysis results to the meshing size. A $1 \mathrm{~nm} \times 1 \mathrm{~nm}$ mesh size generated sufficient accuracy. 


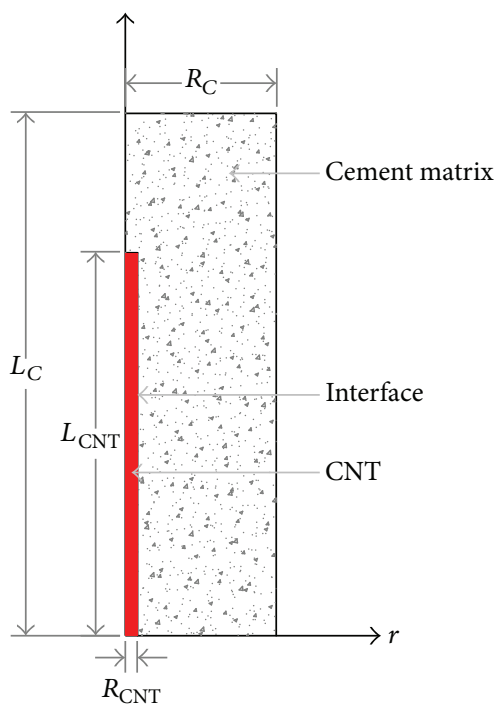

FIgURE 2: Three-phase axisymmetric model for the cement nanocomposite.

2.2.4. Interface. The cohesive surface framework used to describe the CNT/interphase interface can be modeled in Abaqus software as a cohesive element or as a cohesive interaction. In both cases, the interface constitutive response is based on the traction-separation behavior that allows for normal and tangential separation as shown in Figure 3.

The parameters $\delta_{N}$ and $\delta_{T}$ represent the normal separation and tangential sliding, respectively. Modeling the interface as a cohesive interaction instead of a cohesive element could be more appropriate and time saving when the interface thickness is very small or approaching zero and the overall behavior of the composite is investigated rather than the exact stresses within the interface. Because of the cohesive elements and interaction, as the displacement jump across the interface increases, the interface traction increases, reaches a maximum, and then decreases to zero allowing for complete separation (only if the friction between the CNT and the matrix is ignored). The available traction-separation model in Abaqus assumes initially a linearly elastic behavior followed by the initiation and evolution of damage. Once a damage initiation criterion is met, material damage can occur according to specified damage evolution law.

The elastic behavior is written in terms of an elastic constitutive matrix that relates the nominal stresses to the nominal strains across the interface as follows:

$$
t=\left\{\begin{array}{l}
t_{N} \\
t_{T}
\end{array}\right\}=\left(\begin{array}{ll}
K_{N N} & K_{N T} \\
K_{N T} & K_{T T}
\end{array}\right)\left\{\begin{array}{l}
\varepsilon_{N} \\
\varepsilon_{T}
\end{array}\right\},
$$

where $t_{N}$ is normal stress/traction at the interface, $t_{T}$ is tangential stress/traction at the interface, $K_{N N}$ is normal stiffness of the interface, $K_{T T}$ is tangential stiffness of the interface, $K_{N T}$ is coupled stiffness of the interface, $\varepsilon_{N}$ is normal strain at the interface, and $\varepsilon_{T}$ is tangential strain at the interface

Since an uncoupled behavior is desired herein between the normal and shear components, $K_{N T}$ was set equal to zero.
The nominal strains are the separation divided by the original thickness at each integration point. In Abaqus, the default value of the original constitutive thickness is equal to 1.0 if the traction-separation response is specified, which ensures that the nominal strain is equal to the separation at the interface.

When the stresses and/or strains at the interface point satisfy the specified damage initiation criteria, the process of degradation of the response of that point begins. Several damage initiation criteria are available in Abaqus. However, the one used herein depends on the maximum nominal stress criteria, which assumes that the damage has initiated when the maximum nominal stress/traction ratio reaches a value of one. This criterion can be represented by the following equation:

$$
\operatorname{Max}\left\{\frac{\left\langle t_{N}\right\rangle}{t_{N}^{o}}, \frac{t_{T}}{t_{T}^{o}}\right\}=1,
$$

where $t_{N}^{o}$ and $t_{T}^{o}$ represent the peak values of the nominal stress/traction when the deformation is either purely normal or tangential to the interface, respectively. The symbol \langle\rangle used in the discussion below represents the Macaulay bracket which returns only positive values. It is used here to indicate that a pure compressive deformation or stress state does not initiate damage.

The degradation rate of the material stiffness once the corresponding initiation criterion is reached is described by the damage evolution law. The overall material damage is represented by a scalar damage variable $D$, which monotonically changes from 0 to 1 when the loading is further increased after damage initiation. The stress components of the tractionseparation model are affected by the damage according to the following equations:

$$
\begin{array}{ll}
t_{N}= \begin{cases}(1-D) \bar{t}_{N}, & \bar{t}_{N} \geq 0 \\
\bar{t}_{N}, & \text { otherwise (No damage } \\
t_{T}=(1-D) \bar{t}_{T}, & \text { to compressive stiffness) }\end{cases}
\end{array}
$$

where $\bar{t}_{N}$ and $\bar{t}_{T}$ are the stress components predicted by the elastic traction-separation behavior for the current strains without damage. In Abaqus, the damage evolution can be described based on the displacement at failure or based on the fracture energy dissipated as a result of the damage process. The damage softening could be in linear, exponential, or tabular form. A linear damage softening based on the displacement at failure is used herein in which the damage evolution variable $D$ is given by the following expression:

$$
D=\frac{\delta_{m}^{f}\left(\delta_{m}^{\max }-\delta_{m}^{o}\right)}{\delta_{m}^{\max }\left(\delta_{m}^{f}-\delta_{m}^{o}\right)}
$$

where $\delta_{m}=\sqrt{\delta_{N}^{2}+\delta_{T}^{2}}, \delta_{m}^{f}$ is resultant displacement at complete failure, $\delta_{m}^{o}$ is resultant displacement at damage initiation, and $\delta_{m}^{\max }$ is maximum value of the resultant displacement attained during the loading history. 

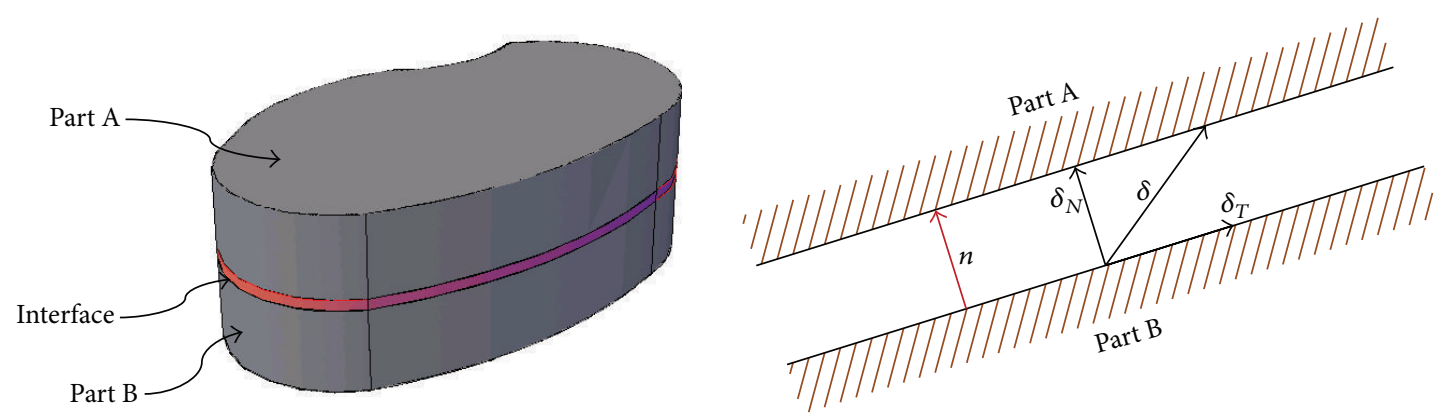

Figure 3: Cohesive interface between two parts.

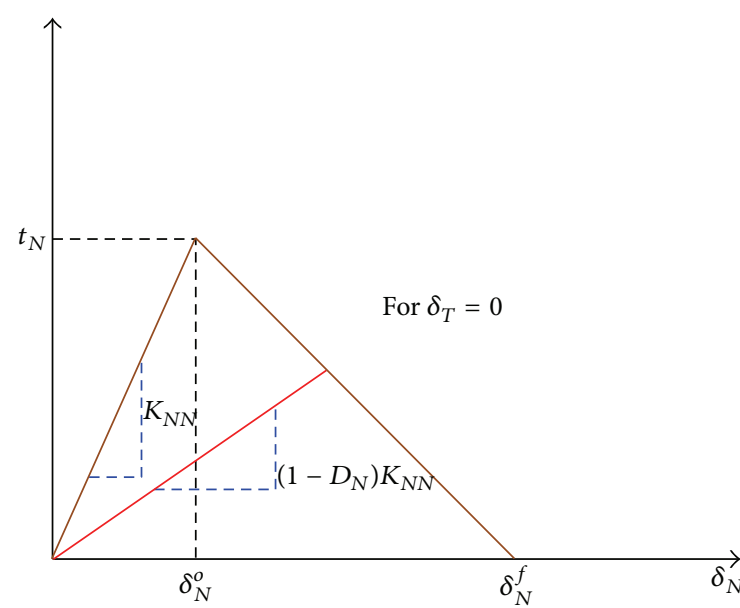

(a)

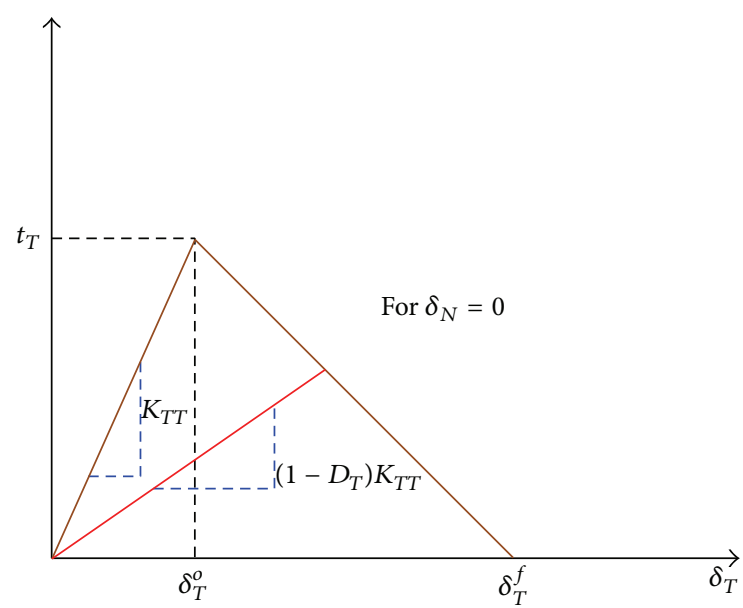

(b)

FIGURE 4: Traction-separation curve for (a) pure normal deformation and (b) pure tangential deformation.

Because the surface of the pristine CNT is very smooth, its friction with the cement matrix after debonding as well as any residual stresses is ignored herein. However, increasing the friction due to the surface treatment of CNTs (e.g., using chemical surface treatment) will be the focus in future work. All stresses in the normal direction were also ignored. Hence, the deformation is assumed to be purely tangential. Therefore, the bond stress-displacement relationship is determined by the interfacial initial stiffness, the interfacial shear strengths, $\mathrm{IS}_{T}$, and the interfacial allowable slip before failure, $\delta^{f}$, or the cohesive energy, $G$, represented by the area under the curve. Typical traction-separation responses with linear damage evolution for pure normal and pure tangential deformations are shown in Figures 4(a) and 4(b), respectively.

In order to investigate the role of the interface while ignoring the interphase, a parametric study was carried out to determine the effect of the interfacial properties on the overall elastic modulus of the nanocomposite cement including the interfacial initial stiffness, $K$, the interfacial shear strengths, $\mathrm{IS}_{T}$, and the interfacial allowable slip before failure, $\delta^{f}$, and the cohesive energy, $G$.

\section{Analytical Method}

The mixture theory provides upper and lower bounds for the composite $E$ value. The upper bound of the composite elastic modulus, which assumes the composite components as acting in parallel, is expressed using the following equation:

$$
E_{\mathrm{Upper}}=f_{\mathrm{CNT}} E_{\mathrm{CNT}}+f_{\mathrm{C}} E_{\mathrm{C}}
$$

The lower bound of the composite elastic modulus is calculated assuming the composite components are acting in series using the following equation:

$$
E_{\mathrm{Lower}}=\frac{E_{\mathrm{CNT}} E_{C}}{f_{\mathrm{C}} E_{\mathrm{CNT}}}+f_{\mathrm{CNT}} E_{C} \text {. }
$$

To validate the proposed axisymmetric model, its obtained $E$ value should lie between the upper and lower bounds of the theory of mixtures.

However, a more realistic assumption is to consider the CNT and the matrix as acting in parallel over the length of the CNT. The CNT-matrix composite is then assumed acting in series with the cement matrix above the CNT (refer to Figure 2).

\section{Parametric Study}

In this parametric study, the interfacial properties were considered and varied for different CNT volume fractions and aspect ratios as follows. 
4.1. Interfacial Shear Strength. The deformation is assumed to be purely tangential and the normal tractions were not considered. Interfacial shear strength values of $1,5,10$, and $20 \mathrm{MPa}$ were investigated. These values did not correspond to any of the values obtained using molecular dynamics (MD) simulations.

4.2. Interfacial Initial Stiffness. The interfacial initial stiffness, $K$, was varied between $1 \times 10^{-8}$ and $5 \times 10^{-8} \mathrm{~N} / \mathrm{nm}^{3}$ with increments of $1 \times 10^{-8} \mathrm{~N} / \mathrm{nm}^{3}$.

4.3. Interfacial Allowable Slip before Failure. The interfacial allowable slip before failure, $\delta^{f}$, represents the CNT displacement out of the surrounding matrix before it is totally debonded and only residual stresses (friction) are acting at the interface. Chan and Andrawes [20] used a relatively conservative approach by assuming a complete detachment at a slip of $0.25 \mathrm{~nm}$, based on thewidth of a hexagonal ring at the surface of a CNT. Slippage values of $0.25 \mathrm{~nm}, 0.50 \mathrm{~nm}$, and $1.00 \mathrm{~nm}$ were considered in this parametric study. By varying the slippage value and keeping other parameters fixed, the cohesive energy, $G$, can be varied and, hence, its effect could be also realized.

4.4. CNT Volume Fraction. CNT volume fractions of $0.1 \%$, $0.3 \%, 0.5 \%$, and $1.0 \%$ were investigated using the interfacial strength values considered in this study. The purpose of varying the CNT volume fraction was to investigate the interaction between its effect and that of the interfacial strength. The allowable slip and the CNT aspect ratio were fixed at $0.25 \mathrm{~nm}$ and 110 , respectively.

In order to be able to compare the modeling results with the experimental results, it is important to convert the volume fraction to the weight fraction (relative to the weight of cement) used in laboratories, the conversion relation could be simply proved, and it is clearly stated in Ghasemzadeh and Jalalabad [24] as follows:

$$
f_{\mathrm{CNT}}=\frac{1}{\left(\left(\rho_{\mathrm{CNT}} / \rho_{\mathrm{C}}\right) \cdot\left(1-w_{\mathrm{CNT}} / w_{\mathrm{CNT}}\right)\right)+1},
$$

where $f_{\mathrm{CNT}}$ : the CNT volume fraction, $w_{\mathrm{CNT}}$ : the CNT weight fraction (by weight of cement), $\rho_{\mathrm{CNT}}$ : the CNT unit weight $\left(\approx 2.1 \mathrm{~g} / \mathrm{cm}^{3}\right), \rho_{\mathrm{C}}$ : the cement matrix unit weight.

Ghasemzadeh and Jalalabad [24] reported value of $2.19 \mathrm{~g} / \mathrm{cm}^{3}$ for the density (unit weight) of cement matrix with 0.45 water/cement ratio (w/c). However, as the $\mathrm{w} / \mathrm{c}$ ratio used in the experimental part of this work is 0.40 and the cement bulk unit weight (including the air voids) is approximately $1.51 \mathrm{~g} / \mathrm{cm}^{3}$, we could estimate the cement paste unit weight by assuming that all voids will be filled with water while mixing. The volume of the cement that is used to prepare the mix will not change but the weight of the cement mixed with water will be 1.4 weight of the bulk cement; hence, the unit weight of the cement matrix (paste) will also be equal to 1.4 multiplied by the unit weight of the bulk cement. Hence, in this study, the unit weight of the cement matrix is taken as $2.11 \mathrm{~g} / \mathrm{cm}^{3}$. Substituting the unit weight values for CNT and cement matrix into (8), CNT volume fraction and weight fraction are approximately equal as follows:

$$
\begin{aligned}
& f_{\mathrm{CNT}}=\frac{1}{\left((2.10 / 2.11) \cdot\left(1-w_{\mathrm{CNT}} / w_{\mathrm{CNT}}\right)\right)+1}, \\
& f_{\mathrm{CNT}}=\frac{1}{\left(\left(1-w_{\mathrm{CNT}}\right) / w_{\mathrm{CNT}}+w_{\mathrm{CNT}} / w_{\mathrm{CNT}}\right)}, \\
& f_{\mathrm{CNT}}=\frac{1}{\left(1 / w_{\mathrm{CNT}}\right)}, \\
& f_{\mathrm{CNT}}=w_{\mathrm{CNT}} .
\end{aligned}
$$

4.5. CNT Aspect Ratio. One advantage for using CNTs is their high aspect ratio (i.e., length over diameter), which is expected to affect positively the composite response. To investigate this effect, aspect ratios of $82.5,110,151,165$, and 173.25 were considered herein. The allowable slip and CNT volume fraction were fixed at $0.25 \mathrm{~nm}$ and $0.5 \%$, respectively.

\section{Analysis, Results, and Discussion}

When addressing the first objective, the elastic modulus values of the cement composite obtained using the $3 \mathrm{D}$ model were compared to those determined using the simplified axisymmetric model. In the parametric study, the interphase layer was ignored and the CNT and cement matrix were assumed perfectly bonded at the interface. Another parametric study was conducted to explore the effect of interfacial properties on the composite elastic modulus by incorporating cohesive surface parameters.

5.1. Comparison between Results of $3 D$ and Axisymmetric Models. In this comparison, it is important to differentiate between the global $X$-, $Y$-, and $Z$-axes in the 3D model and the local $r$-and $z$-axes in the axisymmetric configuration. The local $z$-axis in the axisymmetric problem is assumed to be in the direction of the applied load and hence it corresponds to the global $Z$-axis in the $3 \mathrm{D}$ model. Consequently, the local $r$-axis corresponds to the global $X$ - or $Y$-axes. Two distributions of the straight CNTs (i.e., parallel and inclined with respect to loading) within the cement matrix were investigated for each volume fraction. The curved CNTs could not be modeled in the axisymmetric problem. When the CNTs were randomly distributed parallel to the $Z$-axis (or $z$-axis), the axisymmetric model was simply constructed using one CNT aligned in the $z$-direction and located at the center of the unit cell with the appropriate dimensions. When the CNTs were randomly distributed in arbitrary directions, three distributions were investigated according to the plane in which the fibers were located as follows.

(a) Randomly Distributed Fibers in the Global Y-Z Plane. Different models were constructed using different inclination angles from the $z$-axis ranging between 0 and $\pi / 2$ at $\pi / 12$ interval. Since they are applicable to axisymmetric configuration, inclined CNTs were divided into components in the $r$ and $z$-directions as shown in Figure 5 . The effect of the stress component in the $r$-direction was ignored. This is a valid 


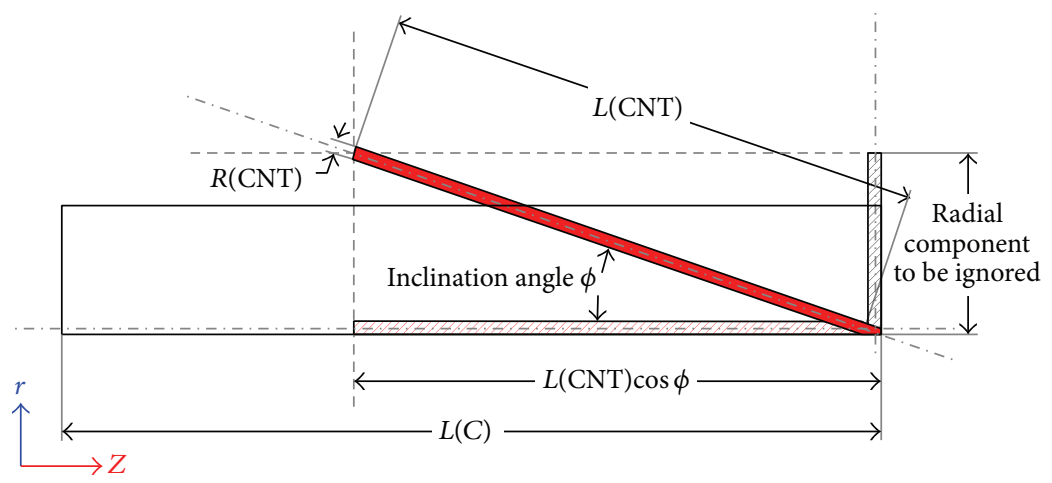

FIgURE 5: Axial and radial CNT components.

TABLE 2: Elastic modulus for different fiber location planes and different inclination angles $(\mathrm{VF}=0.1 \%)$.

\begin{tabular}{lccc}
\hline & \multicolumn{2}{c}{$Y-Z$ and $X-Z$ planes } & $X-Y$ plane \\
& $\begin{array}{c}\text { Inclination angle } \\
\text { from } z \text {-axis (radian) }\end{array}$ & $E(\mathrm{GPa})$ & $E(\mathrm{GPa})$ \\
\hline & $\pi / 12$ & 30.896 & \\
& $\pi / 6$ & 30.862 & \\
& $\pi / 4$ & 30.610 & 30.00 \\
& $\pi / 3$ & 30.411 & \\
& $5 \pi / 12$ & 30.183 & \\
\hline Average $E$ (for the & $\pi / 2$ & 30.000 & \\
distribution in the & & 30.548 & 30.00 \\
specified plane) $(\mathrm{GPa})$ & \multicolumn{3}{|c}{$\mathbf{3 0 . 3 6 6}$} \\
\hline Average $E$ of the \\
composite $(\mathrm{GPa})$ & & \\
\hline
\end{tabular}

assumption because the fibers which are perpendicular to the applied load will not have a significant effect on reinforcing the matrix. This approach eliminates the duplication of the effect of the CNTs in the adjacent unit cells and accounts, to some extent, for the effect of agglomeration within the matrix.

(b) Randomly Distributed Fibers in the Global X-Z Plane. It is the same as Case (a).

(c) Randomly Distributed Fibers in the Global X-Y Plane. Because the CNTs are perpendicular to the applied load, their effect on reinforcing the matrix was ignored.

When the fibers are randomly distributed in the $Z$ direction, the average elastic modulus can be calculated using the following equation:

$$
E_{\mathrm{avr}}=\frac{\sum z}{U / L_{C}},
$$

where $\sum z$ and $U$ are the average stress and total applied displacement in the axial direction, respectively.

When the fibers are randomly distributed in arbitrary directions, a plot was constructed for the average elastic modulus versus the fiber inclination angle from the $z$-axis.
TABLE 3: Elastic modulus for different fiber location planes and different inclination angles $(\mathrm{VF}=0.5 \%)$.

\begin{tabular}{|c|c|c|c|}
\hline & \multicolumn{2}{|c|}{$Y-Z$ and $X-Z$ planes } & \multirow{2}{*}{$\begin{array}{c}X-Y \text { plane } \\
E(\mathrm{GPa})\end{array}$} \\
\hline & $\begin{array}{c}\text { Inclination angle } \\
\text { from } z \text {-axis (radian) }\end{array}$ & $E(\mathrm{GPa})$ & \\
\hline & 0 & 34.312 & \multirow{7}{*}{30.00} \\
\hline & $\pi / 12$ & 34.133 & \\
\hline & $\pi / 6$ & 33.629 & \\
\hline & $\pi / 4$ & 32.854 & \\
\hline & $\pi / 3$ & 31.894 & \\
\hline & $5 \pi / 12$ & 30.846 & \\
\hline & $\pi / 2$ & 30.000 & \\
\hline $\begin{array}{l}\text { Average } E \text { (for the } \\
\text { distribution in the } \\
\text { specified plane) }(\mathrm{GPa})\end{array}$ & & 32.595 & 30.00 \\
\hline $\begin{array}{l}\text { Average } E \text { of the } \\
\text { composite }(\mathrm{GPa})\end{array}$ & \multicolumn{3}{|c|}{31.730} \\
\hline
\end{tabular}

The elastic modulus values were obtained using (10) while the fiber inclination angle was varied from 0 to $\pi / 2$. The area under the curve was computed and then divided by $\pi / 2$ to get the average matrix elastic modulus for each case. Tables 2 and 3 summarize the values of the composite elastic modulus for different inclination angles about the $z$-axis. The plots for the $0.1 \%$ and $0.5 \%$ volume fractions are shown in Figures $6(\mathrm{a})$ and $6(\mathrm{~b})$, respectively.

The value of the elastic modulus of the cement nanocomposite was assumed to be equal to that of the matrix, that is, $30 \mathrm{GPa}$ when the fibers were distributed in the global $X-Y$.

Finally, in order to compute the average composite elastic modulus when the fibers are distributed randomly in any direction, it is important to assume rough percentages of the fibers distributed in the $Y-Z, X-Z$, and $X-Y$ planes. For example, assuming that the fibers are distributed in equal percentages in all planes (i.e., 33\% in each plane), the elastic modulus for the $0.1 \%$ and $0.5 \%$ CNT volume fractions is $30.366 \mathrm{GPa}$ and $31.73 \mathrm{GPa}$, respectively. These values are in good agreement with those obtained using the $3 \mathrm{D}$ simulation.

Table 4 summarizes the comparison between the elastic modulus values obtained using the $3 \mathrm{D}$, axisymmetric, and mixture theory models. 


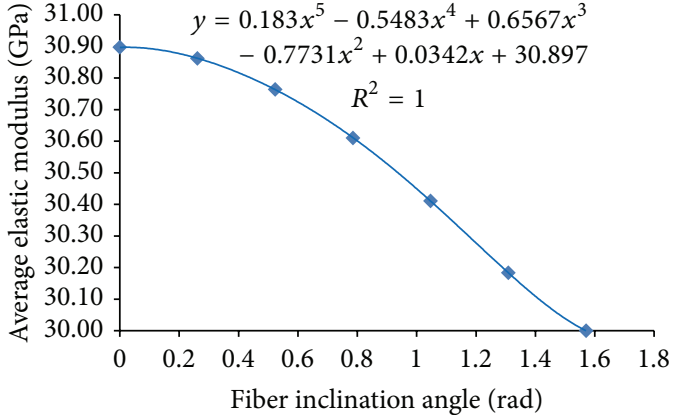

(a)

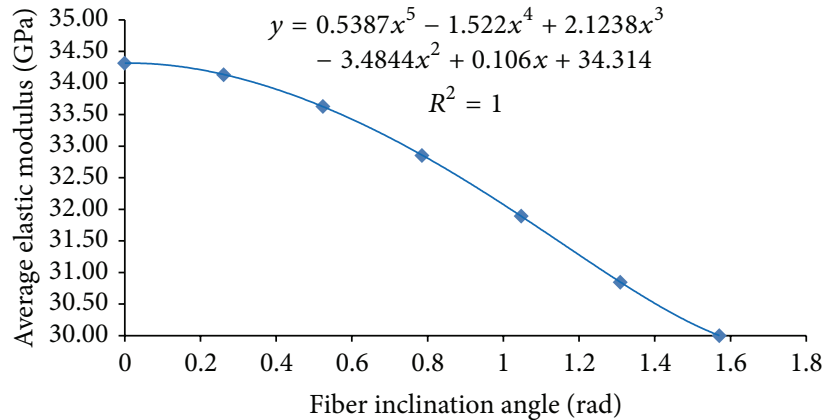

(b)

Figure 6: Plot of the average elastic modulus versus fiber inclination angle about $z$-axis. (a) VF $=0.1 \%$ and $(b) \mathrm{VF}=0.5 \%$.

TABLE 4: Elastic modulus for 3D and axisymmetric models.

\begin{tabular}{|c|c|c|c|c|c|c|c|}
\hline & \multicolumn{2}{|c|}{ Straight, $Z$-direction } & \multicolumn{2}{|c|}{ Straight, random } & \multicolumn{2}{|r|}{ Curved } & \multirow{2}{*}{$\begin{array}{l}\text { Mixture theory } \\
\text { (upper bound) }\end{array}$} \\
\hline & 3D model & Axisymmetric model & $3 \mathrm{D}$ model & Axisymmetric model & 3D model & Axisymmetric model & \\
\hline \multicolumn{8}{|l|}{$\mathrm{VF}=0.1 \%$} \\
\hline$E(\mathrm{GPa})$ & 31.293 & 30.896 & 30.635 & 30.366 & 30.579 & N.A & 30.97 \\
\hline$\%$ increase & 4.31 & 3.00 & 2.12 & 1.22 & 1.93 & l & 3.23 \\
\hline \multicolumn{8}{|l|}{$\mathrm{VF}=0.5 \%$} \\
\hline$E(\mathrm{GPa})$ & 34.915 & 34.312 & 31.691 & 31.73 & 31.535 & N.A & 34.85 \\
\hline$\%$ increase & 16.38 & 14.37 & 5.64 & 5.77 & 5.12 & l & 16.17 \\
\hline
\end{tabular}

${ }^{*}$ N.A: not applicable.

As shown in Table 4, the predicted elastic modulus values using the axisymmetric model for both volume fractions are in good agreement with those obtained using the $3 \mathrm{D}$ model when the CNTs are straight and aligned in the longitudinal direction in which the load is applied. The results are also very close to the upper bound of the mixture theory, which assumes that the composite components are acting completely in parallel. The proposed method for approximating the elastic modulus using the axisymmetric model gives acceptable results when the CNTs are straight and randomly aligned in arbitrary directions. It considers the alignment of the fibers in three Cartesian planes and ignores the effect of the fiber component perpendicular to the applied load on the elastic modulus of the nanocomposite. This approach may account, to some extent, for the agglomeration of the fibers by neglecting the effect of radial component of the CNT. On the other hand, the axisymmetric model is not applicable for the case of the curved CNTs. However, the 3D model results show that the elastic modulus is reduced to almost the same degree for straight randomly aligned CNTs and curved randomly dispersed CNTs for the small volume fractions that were investigated. Hence, the elastic modulus values obtained using the axisymmetric model for randomly aligned straight CNTs can be, conservatively, used to estimate those for the curved ones for small volume fractions. However, this may not be the case if the CNT volume fraction is higher. This should be investigated using the $3 \mathrm{D}$ model.

5.2. Parametric Study on the Effect of Interfacial Properties (with No Interphase). The individual roles of the key parameters describing the cohesive surface used to model the interface were studied. It is worth noting that the effect of friction between the CNT and the cement matrix after debonding was ignored as shown in the bond-displacement relationship in Figures 4(a) and 4(b). All normal stresses were also ignored and the deformations were assumed to be purely tangential. The parametric characterization was previously illustrated in Section 4. The following subsections will present and discuss the results of the effect of each individual parameter as well as the interaction of those effects with the CNT volume fractions and aspect ratios. The computations were performed by using a constant displacement rate until the axial strain reaches a strain value of 0.0005 .

\subsubsection{Effect of Interfacial Strength on the Stress-Strain Curve.} When the CNT and cement matrix are assumed to be perfectly bonded, the stress-strain diagram is perfectly linear. However, by incorporating the cohesive surface of the interface, the stress-strain diagram tends to be nonlinear. The composite elastic modulus is computed as the slope of the best line fit. However, loading (up to the specified strain) and unloading were carried out to investigate the stressstrain nonlinearity due to the interfacial debonding. The secant modulus (which may be referred to as damaged elastic modulus) was computed. Figure 7 shows the relationship between the damaged elastic modulus and the corresponding strain for different interfacial shear strength values. It is worth noting that the interfacial initial stiffness, allowable slip before failure, CNT volume fraction, and aspect ratio were fixed at $1 \times 10^{-8} \mathrm{~N} / \mathrm{nm}^{3}, 0.25 \mathrm{~nm}, 0.5 \%$, and 110 , 


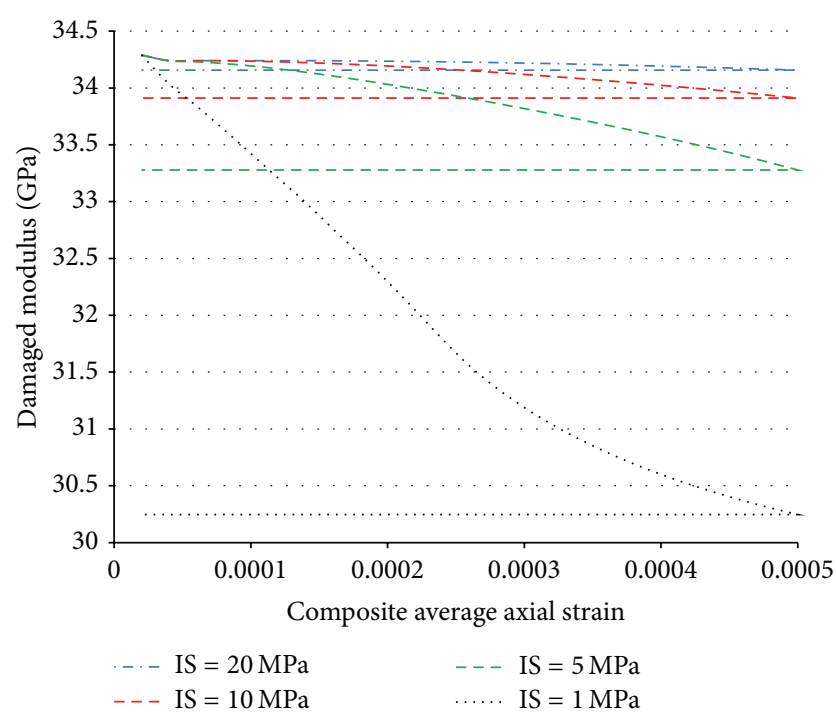

FIGURE 7: Composite damaged modulus versus average axial strain $\left(K=1 \times 10^{-8} \mathrm{~N} / \mathrm{nm}^{3}, \mathrm{VF}=0.50 \%\right.$, allowable slip $=0.25 \mathrm{~nm}$, and $\mathrm{CNT}$ aspect ratio $=110$ ).

TABLE 5: Elastic modulus for different interfacial strengths and different interfacial allowable slips $\left(K=1 \times 10^{-8} \mathrm{~N} / \mathrm{nm}^{3}, \mathrm{VF}=0.5 \%\right.$, and aspect ratio $=110$ ).

\begin{tabular}{lccc}
\hline Interfacial shear strength & \multicolumn{3}{c}{ Allowable slip (nm) } \\
& 0.25 & 0.5 & 1 \\
\hline Perfectly bonded & & 34.312 & \\
$\quad$ Model & & 34.579 & \\
$\quad$ Analytical & 34.193 & 34.194 & 34.195 \\
IS $=20 \mathrm{MPa}$ & 34.033 & 34.042 & 34.046 \\
IS $=10 \mathrm{MPa}$ & 33.598 & 33.645 & 33.664 \\
IS $=5 \mathrm{MPa}$ & 30.836 & 31.159 & 31.293 \\
IS $=1 \mathrm{MPa}$
\end{tabular}

respectively. The results show that the degradation rate of the elastic modulus is higher for lower values of interfacial shear strength. This degradation is followed by a plateau, which represents the elastic modulus at the unloading stage.

\subsubsection{Effect of Interfacial Strength and Allowable Slip (Cohe-} sive Energy). Table 5 shows the predicted composite elastic modulus for different interfacial strengths and allowable slips. The results were obtained for an interfacial initial stiffness of $1 \times 10^{-8} \mathrm{~N} / \mathrm{nm}^{3}$, a CNT volume fraction of $0.50 \%$, and a CNT aspect ratio of 110 .

The results show that the $E$ values obtained using axisymmetric model for the perfectly bonded case are close to those computed analytically by assuming the CNT and the matrix acting in parallel over the length of the CNT and in series with the portion of the cement matrix above the CNT. In the region over the length of the CNT, the CNT volume fraction is equal to 0.00682 . In this region, the effective elastic modulus is equal to $36.614 \mathrm{GPa}$ (in parallel action). The region, which includes the $\mathrm{CNT}$ and the matrix along CNT

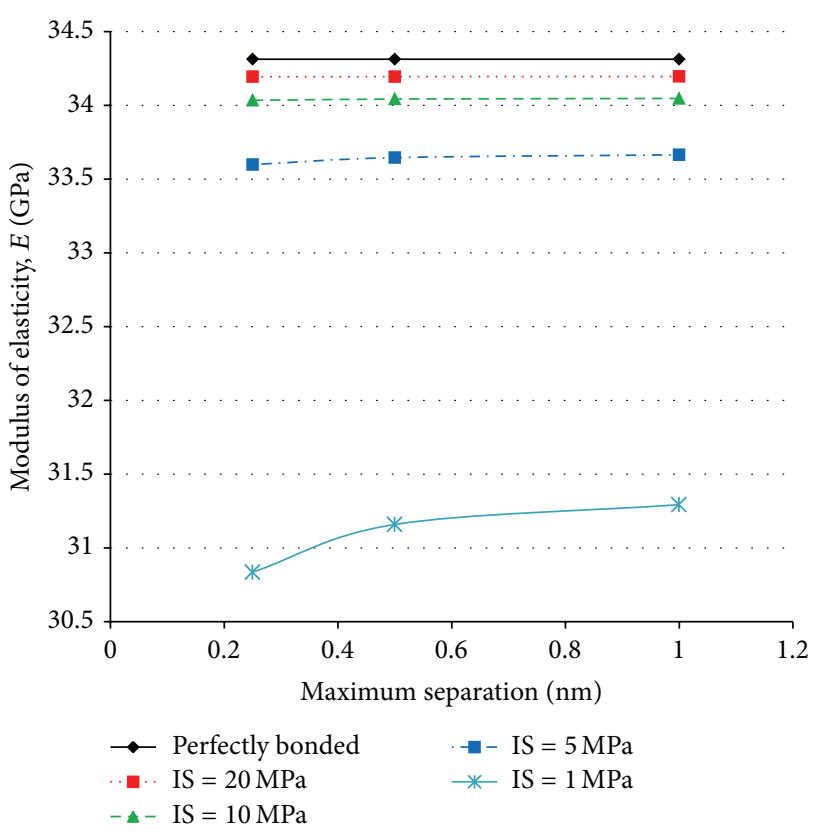

FIGURE 8: Average elastic modulus versus interfacial allowable slip for different interfacial strength values $(\mathrm{VF}=0.5 \%$, aspect ratio $=$ $110)$.

length, has a volume fraction of 0.733 acting in series with another region and 0.267 volume fraction of cement matrix above the CNT to give average composite elastic modulus of $34.579 \mathrm{GPa}$. Figure 8 shows the variation of the composite elastic modulus with the allowable slip for different values of interfacial strengths. Figure 8 shows the variation of the composite elastic modulus with the allowable slip for different values of interfacial strengths.

Figure 8 shows that, by keeping the interface strength constant, the elastic modulus of the nanocomposite increases with increasing maximum allowable separation before failure. Initially, the elastic modulus increases at a faster rate. Then, the rate of increase starts to decrease after a certain separation value. The increase rate of the elastic modulus decreases with increasing interfacial strength. Figure 9 shows the variation of the elastic modulus with the interfacial strength for different allowable slip values.

Figure 9 shows that, for a particular allowable separation value, the modulus of the nanocomposite increases with increasing interface strength. It also shows that, after a certain interfacial strength, the curves for different allowable separation values become very close to each other until they intersect. This means that the impact of increasing the allowable separation is reduced for higher interfacial strength values. This may be related to the effect of cohesive energy. As the interfacial strength becomes higher, the increase in the cohesive energy value needed for debonding the CNT from the matrix (i.e., area under the traction-displacement diagram) for a certain allowable separation value is larger. This may explain that, after a certain interfacial strength value, the change of the material properties including the elastic modulus is slower as the cohesive energy needed for 
TABLE 6: Elastic modulus for different interfacial initial stiffness strengths (allowable slip $=0.25 \mathrm{~nm}, \mathrm{VF}=0.5 \%$, and aspect ratio $=110$ ).

\begin{tabular}{lcccc}
\hline Interfacial shear strength & \multicolumn{3}{c}{$K\left(\mathrm{~N} / \mathrm{nm}^{3}\right)$} \\
& $1 * 10^{-8}$ & $2 * 10^{-8}$ & $3 * 10^{-8}$ & $4 * 10^{-8}$ \\
\hline IS $=10 \mathrm{MPa}$ & 34.033 & 34.038 & 34.04 & 34.041 \\
\hline
\end{tabular}

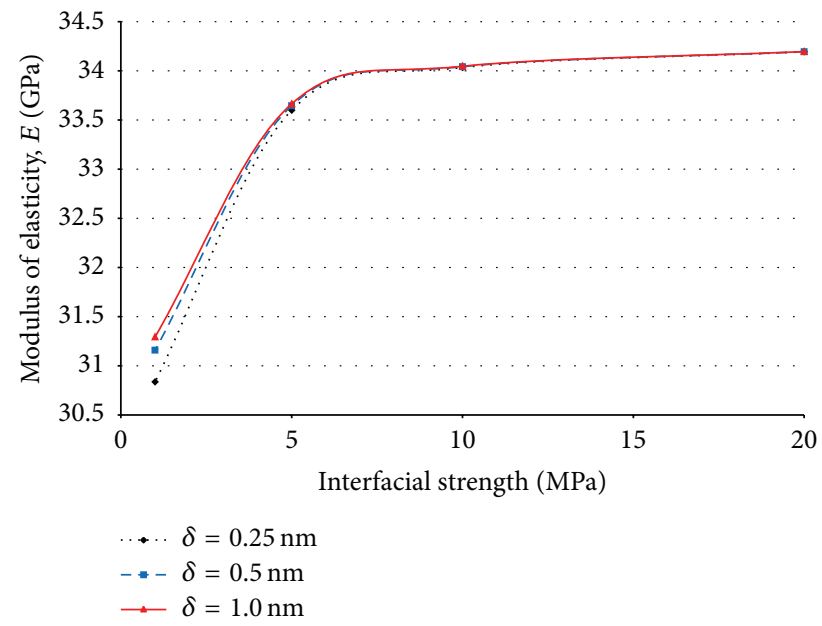

Figure 9: Average elastic modulus versus interfacial strengths for different interfacial allowable slip values $(\mathrm{VF}=0.5 \%$, aspect ratio $=$ $110)$.

TABLE 7: Elastic modulus for different interfacial strengths and CNT volume fractions $\left(K=1 \times 10^{-8} \mathrm{~N} / \mathrm{nm}^{3}\right.$, allowable slip $=0.25 \mathrm{~nm}$, and aspect ratio $=110)$.

\begin{tabular}{lcccc}
\hline \multirow{2}{*}{ Interfacial shear strength } & \multicolumn{5}{c}{ CNT volume fraction (\%) } \\
& 0.1 & 0.3 & 0.5 & 1 \\
\hline Perfectly bonded & & & & \\
$\quad$ Model & 30.896 & 32.639 & 34.312 & 38.205 \\
$\quad$ Analytical & 30.958 & 32.810 & 34.579 & 38.675 \\
IS $=20 \mathrm{MPa}$ & 30.873 & 32.569 & 34.193 & 37.956 \\
IS $=10 \mathrm{MPa}$ & 30.843 & 32.475 & 34.033 & 37.628 \\
IS $=5 \mathrm{MPa}$ & 30.754 & 32.211 & 33.598 & 36.791 \\
IS $=1 \mathrm{MPa}$ & 30.166 & 30.499 & 30.836 & 31.692 \\
\hline
\end{tabular}

debonding (i.e., responsible for properties change) becomes higher as shown in Figure 9.

5.2.3. Effect of Interfacial Initial Stiffness. Table 6 summarizes the predicted composite elastic modulus for different initial interfacial stiffness given interfacial shear strength of $10 \mathrm{MPa}$, an allowable slip of $0.25 \mathrm{~nm}$, a CNT volume fraction of $0.50 \%$, and a CNT aspect ratio of 110 .

It is obvious that changing the initial interfacial stiffness will not significantly change the composite elastic modulus and hence the effect of this parameter is minor.

5.2.4. Interaction between the Effects of Interfacial Strength and CNT Volume Fraction. Table 7 summarizes the predicted composite elastic modulus using different interfacial

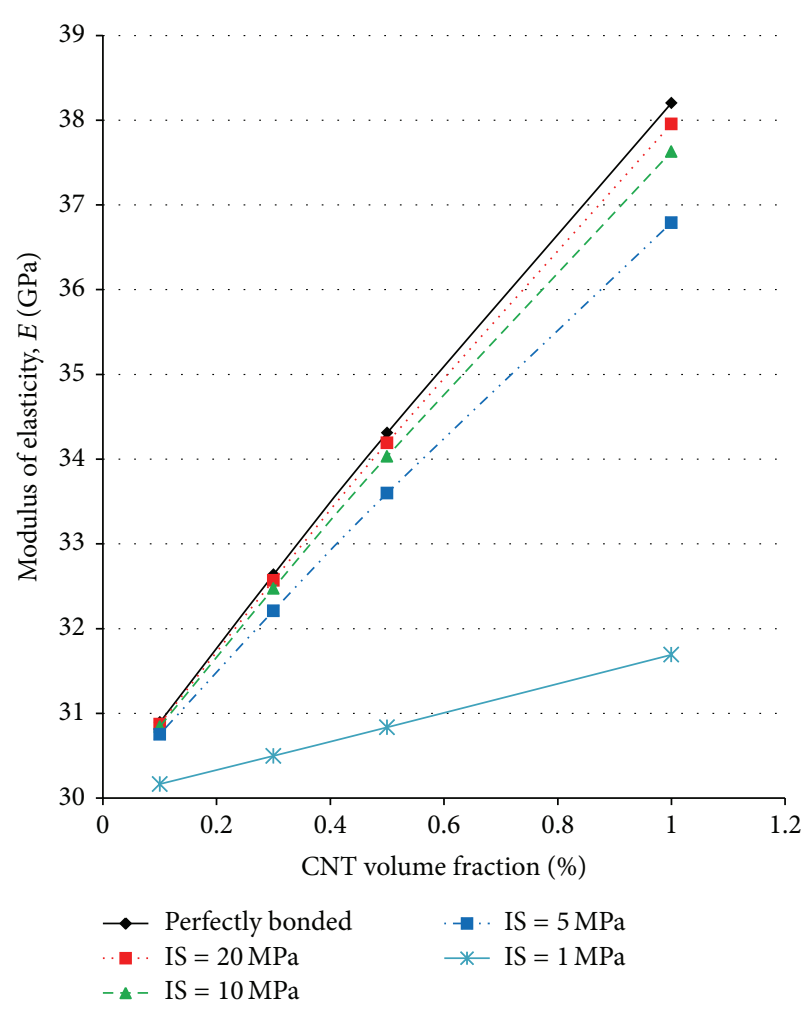

FIGURE 10: Average elastic modulus versus CNT volume fraction for different interfacial strength values $\left(K=1 \times 10^{-8} \mathrm{~N} / \mathrm{nm}^{3}\right.$, allowable slip $=0.25 \mathrm{~nm}$, and aspect ratio $=110)$.

strengths and CNT volume fractions given an interfacial initial stiffness of $1 \times 10^{-8} \mathrm{~N} / \mathrm{nm}^{3}$, an allowable slip of $0.25 \mathrm{~nm}$, and an aspect ratio of $110 \mathrm{CNT}$. For the perfectly bonded case, the values obtained using the model are in good agreement with those computed analytically as described in Section 3.

Figure 10 shows the variation of the composite elastic modulus versus CNT volume fraction for different interfacial strength values.

Figure 10 shows that, by keeping the interface strength constant, the elastic modulus of the nanocomposite increases with increasing CNT volume fraction for the range of the volume fractions used in this study. The rate of increase is larger for higher interface strengths.

Figure 11 shows the variation of the elastic modulus with the interfacial strength for different CNT volume fraction values.

Figure 11 shows that, for a particular CNT volume fraction value, the modulus of the nanocomposite increases with increasing interface strength. Initially, the elastic modulus increases at a faster rate and then decreases after a certain 


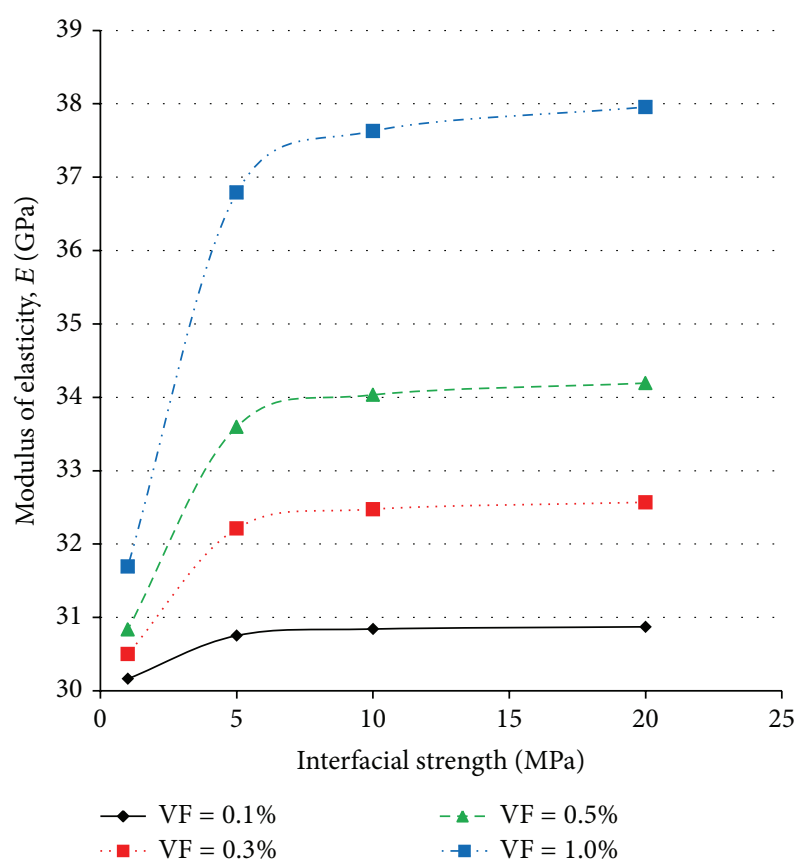

FIGURE 11: Average elastic modulus versus interfacial strengths for different CNT volume fraction values of $\left(K=1 \times 10^{-8} \mathrm{~N} / \mathrm{nm}^{3}\right.$, allowable slip $=0.25 \mathrm{~nm}$, and aspect ratio $=110$ ).

TABLE 8: Elastic modulus for different interfacial strengths and CNT aspect ratios $\left(K=1 \times 10^{-8} \mathrm{~N} / \mathrm{nm}^{3}\right.$, allowable separation $=0.25 \mathrm{~nm}$, and $\mathrm{VF}=0.50 \%)$.

\begin{tabular}{lccccc}
\hline \multirow{2}{*}{ Interfacial shear strength } & \multicolumn{5}{c}{ CNT aspect ratio } \\
& 82.7 & 110 & 151 & 165 & 173.25 \\
\hline $\begin{array}{l}\text { Perfectly bonded } \\
\quad \text { Model }\end{array}$ & 34.066 & 34.312 & 34.564 & 34.619 & 34.695 \\
$\quad$ Analytical & 34.394 & 34.579 & 34.778 & 34.818 & 34.85 \\
IS $=20 \mathrm{MPa}$ & 33.923 & 34.193 & 34.47 & 34.53 & 34.569 \\
IS $=10 \mathrm{MPa}$ & 33.73 & 34.033 & 34.346 & 34.414 & 34.432 \\
IS $=5 \mathrm{MPa}$ & 33.207 & 33.598 & 34.009 & 34.099 & 34.142 \\
IS $=1 \mathrm{MPa}$ & 30.666 & 30.836 & 31.089 & 31.149 & 31.179 \\
\hline
\end{tabular}

interfacial strength value. For small CNT volume fraction values, the rate of increase in the modulus of the nanocomposite with interfacial strength is smaller.

5.2.5. Interaction between the Effect of Interfacial Shear Strength and CNT Aspect Ratio. Table 8 shows the predicted composite elastic modulus for different interfacial strengths and CNT aspect ratio fractions for $1 \times 10^{-8} \mathrm{~N} / \mathrm{nm}^{3}$ interfacial initial stiffness, $0.25 \mathrm{~nm}$ allowable slip, and $0.50 \% \mathrm{CNT}$ volume fraction. For the perfectly bonded case, the values resulting from the model are shown to be in good agreement with the values computed analytically.

Figure 12 shows the variation of the composite elastic modulus with the CNT aspect ratio for different interfacial strength values.

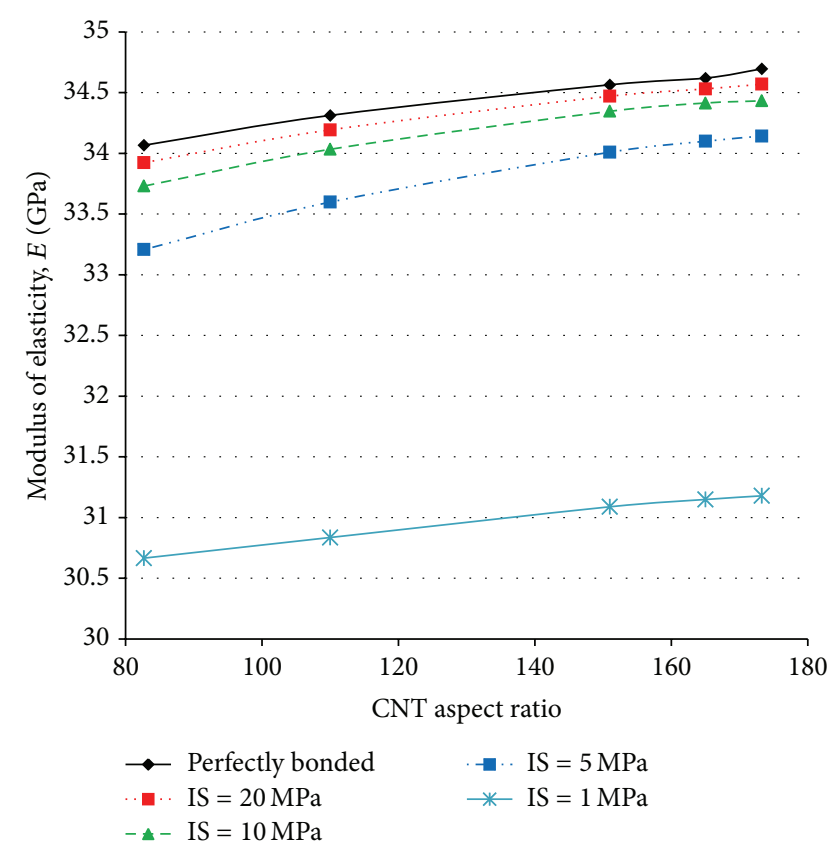

FIGURE 12: Plots of the average elastic modulus versus CNT aspect ratio for different values of interfacial strengths $\left(K=1 \times 10^{-8} \mathrm{~N} / \mathrm{nm}^{3}\right.$, allowable slip $=0.25 \mathrm{~nm}$, and $\mathrm{VF}=0.50 \%$ ).

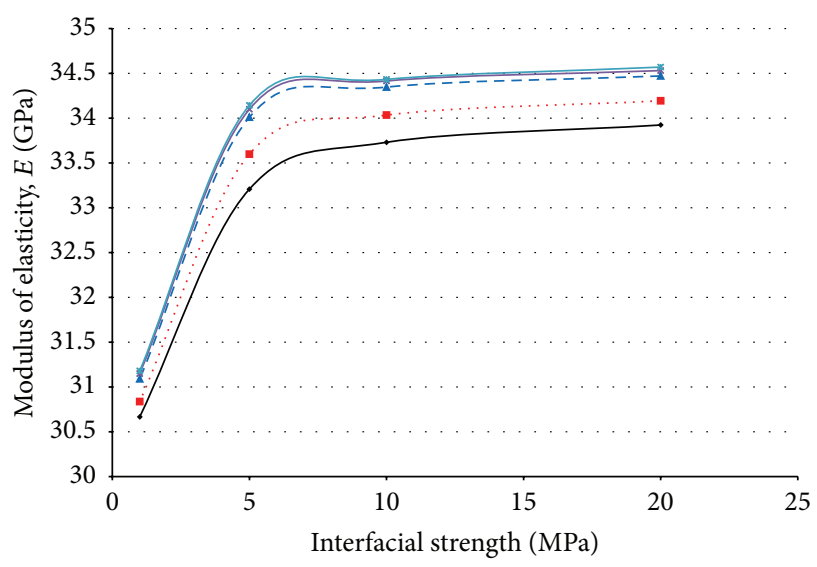

$$
\begin{array}{rlll}
- \text { Aspect ratio } & =82.7 \quad \rightarrow \text { Aspect ratio }=165 \\
\text { - Aspect ratio } & =110 \quad \text { * } \\
\text { - } & \text { Aspect ratio }=151
\end{array}
$$

FIGURE 13: Average elastic modulus versus interfacial strengths for different CNT aspect ratio values $\left(K=1 \times 10^{-8} \mathrm{~N} / \mathrm{nm}^{3}\right.$, allowable slip $=0.25 \mathrm{~nm}$, and $\mathrm{VF}=0.50 \%$ ).

Figure 12 shows that, for particular interface strength, the elastic modulus of the nanocomposite increases with increasing the CNT aspect ratio. The rate of increase decreases with increasing aspect ratios. The rate of increase is also larger for small interface strengths.

Figure 13 shows the variation of the elastic modulus with the interfacial strength for different CNT aspect ratio values.

Figure 13 shows that, for a particular CNT aspect ratio value, the modulus of the nanocomposite increases with increasing interface strength. Initially, the elastic modulus 
increases at a faster rate and becomes very small after a certain interfacial strength value. The rate of increase is larger for smaller values of CNT aspect ratio.

\section{Conclusions}

This paper investigates the elasticity of CNT-reinforced cement composite. 3D and axisymmetric models were developed and their results were compared. The effect of the interface properties on the elastic response of cement nanocomposite was also studied using the axisymmetric model. The interactions between their effect and those of other parameters such as the CNT volume fraction and aspect ratio were also explored. The following conclusions can be made.

(i) The predicted elastic modulus values obtained using the axisymmetric model are in good agreement with those obtained using the 3D model when CNTs were assumed straight and aligned in the loading direction. The results are also very close to the upper bound values obtained using the mixture theory of mixtures which assumes composite.

(ii) When the CNTs are straight and randomly aligned in arbitrary directions, the predicted elastic modulus values obtained using the axisymmetric model are acceptable. The model considers the alignment of the fibers in the three Cartesian planes and ignores the effect of the fiber component perpendicular to the applied load. This may account, to some extent, for the agglomeration of the fibers by neglecting the effect of radial component of CNTs.

(iii) The axisymmetric model cannot be used when curved CNTs are assumed. However, it could be noted from the 3D model results that the elastic modulus is reduced to almost the same degree for straight randomly aligned CNTs and curved randomly dispersed CNTs for the small volume fractions which were investigated. Hence, the elastic modulus values obtained using the axisymmetric model for randomly aligned straight CNTs can be, conservatively, used to estimate those for the curved ones for small volume fractions. However, this may not be the case if the CNT volume fraction is higher. This should be investigated using the 3D model.

(iv) The composite elastic modulus increases with increasing interfacial strength for the same CNT volume fraction, CNT aspect ratio, and CNT and cement matrix mechanical properties. The rate of increase is initially fast and becomes very small after a certain interfacial strength value.

(v) The strain value at which the damage between the CNT and matrix initiates is larger for higher interfacial strength values.

(vi) The impact of increasing the allowable separation becomes smaller for higher interfacial strength values. (vii) After a certain allowable separation value, the rate of increase of the composite elastic modulus becomes very small for all interfacial strength values.

(viii) The composite elastic modulus increases with increasing CNT volume fraction for a particular interfacial strength. The rate of increase is larger for higher interface strengths.

(ix) The effect of interfacial strength becomes more significant as the CNT volume fraction increases.

(x) The elastic modulus of the nanocomposite increases with increasing CNT aspect ratio for a particular interfacial strength. The rate of increase is larger for small interface strengths. However, the composite elastic modulus will not be significantly affected when the length of the CNT equals the length of the matrix.

Future experimental work may be directed towards investigating the effect of the interfacial transition zone properties on the composite mechanical properties and also towards modeling the behavior of the cement matrix using a plastic damaged model.

\section{Disclaimer}

The statements made herein are solely the responsibility of the authors.

\section{Conflict of Interests}

The authors declare that there is no conflict of interests regarding the publication of this paper.

\section{Acknowledgment}

This work was made possible by a National Priority Research Program award (NPRP 4-1142-2-440) from the Qatar National Research Fund (a member of The Qatar Foundation).

\section{References}

[1] S. Iijima, "Helical microtubules of graphitic carbon," Nature, vol. 354, no. 6348, pp. 56-58, 1991.

[2] E. T. Thostenson, Z. F. Ren, and T.-W. Chou, "Advances in the science and technology of carbon nanotubes and their composites: a review," Composites Science and Technology, vol. 61, no. 13, pp. 1899-1912, 2001.

[3] P. G. Collins and P. Avouris, "Nanotubes for electronics," Scientific American, vol. 283, no. 6, pp. 38-45, 2000.

[4] A. A. Ensafi, H. Karimi-Maleh, S. Mallakpour, and M. Hatami, "Simultaneous determination of $\mathrm{N}$-acetylcysteine and acetaminophen by voltammetric method using $N$-(3,4-dihydroxyphenethyl)-3,5-dinitrobenzamide modified multiwall carbon nanotubes paste electrode," Sensors and Actuators B: Chemical, vol. 155, no. 2, pp. 464-472, 2011. 
[5] A. A. Ensafi, H. Karimi-Maleh, S. Mallakpour, and B. Rezaei, "Highly sensitive voltammetric sensor based on catecholderivative-multiwall carbon nanotubes for the catalytic determination of captopril in patient human urine samples," Colloids and Surfaces B: Biointerfaces, vol. 87, no. 2, pp. 480-488, 2011.

[6] T. Tavana, M. A. Khalilzadeh, H. Karimi-Maleh, A. A. Ensafi, H. Beitollahi, and D. Zareyee, "Sensitive voltammetric determination of epinephrine in the presence of acetaminophen at a novel ionic liquid modified carbon nanotubes paste electrode," Journal of Molecular Liquids, vol. 168, pp. 69-74, 2012.

[7] A. Mokhtari, H. Karimi-Maleh, A. A. Ensafi, and H. Beitollahi, "Application of modified multiwall carbon nanotubes paste electrode for simultaneous voltammetric determination of morphine and diclofenac in biological and pharmaceutical samples," Sensors and Actuators, B: Chemical, vol. 169, pp. 96-105, 2012.

[8] R. Moradi, S. A. Sebt, H. Karimi-Maleh et al., "Synthesis and application of FePt/CNTs nanocomposite as a sensor and novel amide ligand as a mediator for simultaneous determination of glutathione, nicotinamide adenine dinucleotide and tryptophan," Physical Chemistry Chemical Physics, vol. 15, no. 16, pp. 5888-5897, 2013.

[9] M. Najafi, M. A. Khalilzadeh, and H. Karimi-Maleh, "A new strategy for determination of bisphenol $\mathrm{A}$ in the presence of Sudan I using a $\mathrm{ZnO} / \mathrm{CNTs} /$ ionic liquid paste electrode in food samples," Food Chemistry, vol. 158, pp. 125-131, 2014.

[10] H. M. Hsiao and I. M. Daniel, "Elastic properties of composites with fiber waviness," Composites Part A: Applied Science and Manufacturing, vol. 27, no. 10, pp. 931-941, 1996.

[11] F. T. Fisher, R. D. Bradshaw, and L. C. Brinson, "Fiber waviness in nanotube-reinforced polymer composites-I: modulus predictions using effective nanotube properties," Composites Science and Technology, vol. 63, no. 11, pp. 1689-1703, 2003.

[12] R. D. Bradshaw, F. T. Fisher, and L. C. Brinson, "Fiber waviness in nanotube-reinforced polymer composites-II: modeling via numerical approximation of the dilute strain concentration tensor," Composites Science and Technology, vol. 63, no. 11, pp. 1705-1722, 2003.

[13] D.-L. Shi, X.-Q. Feng, Y. G. Y. Huang, K.-C. Hwang, and H. Gao, "The effect of nanotube waviness and agglomeration on the elastic property of carbon nanotube-reinforced composites," Journal of Engineering Materials and Technology, vol. 126, no. 3, pp. 250-257, 2004.

[14] L. H. Shao, R. Y. Luo, S. L. Bai, and J. Wang, "Prediction of effective moduli of carbon nanotube-reinforced composites with waviness and debonding," Composite Structures, vol. 87, no. 3, pp. 274-281, 2009.

[15] P. D. Spanos and M. Esteva, "Effect of stochastic nanotube waviness on the elastic and thermal properties of nanocomposites by fiber embedment in finite elements," Journal of Computational and Theoretical Nanoscience, vol. 6, no. 10, pp. 2317-2333, 2009.

[16] A. Ranjbaran, "Embedding of reinforcements in reinforced concrete elements implemented in DENA," Computers \& Structures, vol. 40, no. 4, pp. 925-930, 1991.

[17] B. M. Tyson, R. K. Abu Al-Rub, A. Yazdanbakhsh, and Z. Grasley, "Carbon nanotubes and carbon nanofibers for enhancing the mechanical properties of nanocomposite cementitious materials," Journal of Materials in Civil Engineering, vol. 23, no. 7, pp. 1028-1035, 2011.

[18] N. Chandra, Cohesive Zone Approach to Multiscale Modeling of Nanotube Reinforced Composites, Final Report, Florida A\&M University, 2007.
[19] A. E. Naaman, G. G. Namur, J. M. Alwan, and H. S. Najm, "Fiber pullout and bond slip. II: Experimental validation," Journal of Structural Engineering, vol. 117, no. 9, pp. 2791-2800, 1991.

[20] L. Y. Chan and B. Andrawes, "Characterization of the uncertainties in the constitutive behavior of carbon nanotube/cement composites," Science and Technology of Advanced Materials, vol. 10, no. 4, Article ID 045007, pp. 1-13, 2009.

[21] L. Chan and B. Andrawes, "Numerical modeling of flexural enhancement in carbon nanotube/cement composite," in Structures Congress 2009, pp. 1-8, 2009.

[22] F. Dalmas, R. Dendievel, L. Chazeau, J.-Y. Cavaillé, and C. Gauthier, "Carbon nanotube-filled polymer composites. Numerical simulation of electrical conductivity in three-dimensional entangled fibrous networks," Acta Materialia, vol. 54, no. 11, pp. 2923-2931, 2006.

[23] A. H. Tehrani and R. K. Abu Al-Rub, "Mesomechanical modeling of polymerclay nanocomposites using a viscoelasticviscoplastic-viscodamage constitutive model," Journal of Engineering Materials and Technology, vol. 133, no. 4, Article ID 041011, 8 pages, 2011.

[24] H. Ghasemzadeh and E. A. Jalalabad, "Computing the compressive strength of carbon nanotube/cement composite," International Journal of Civil Engineering, vol. 9, no. 3, pp. 223-229, 2011. 

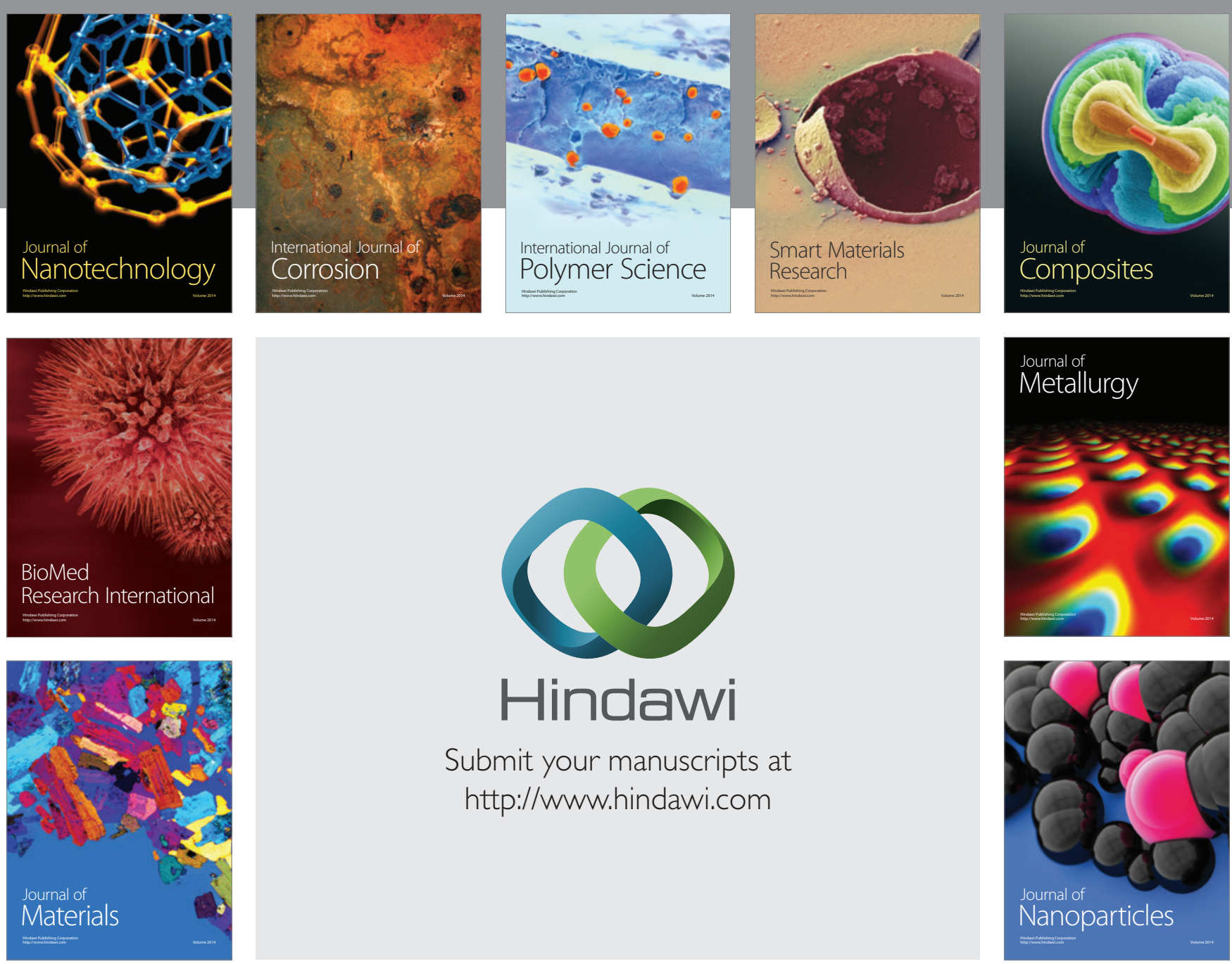

Submit your manuscripts at http://www.hindawi.com
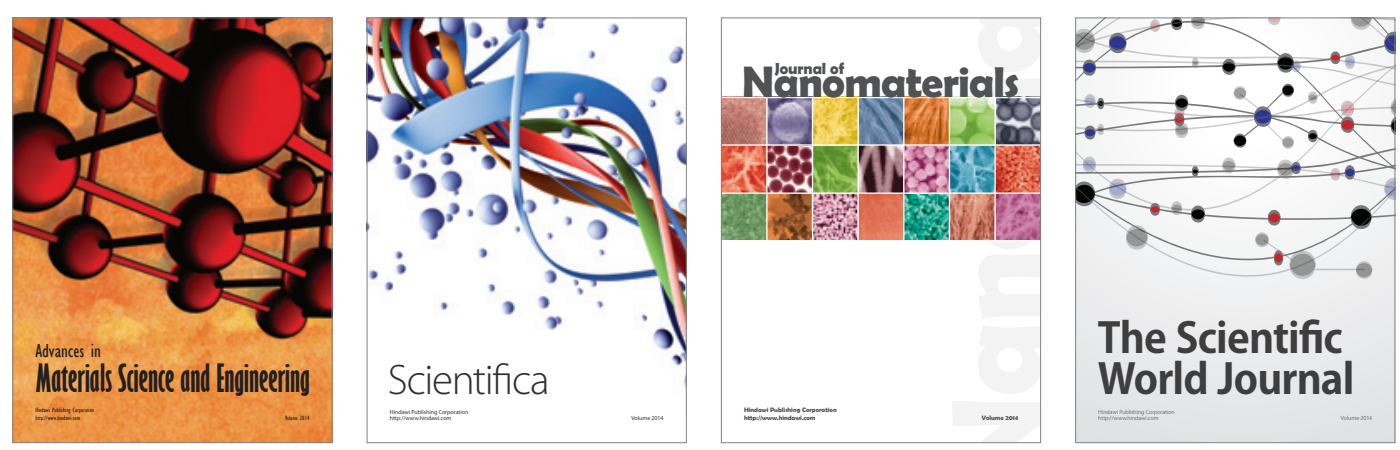

\section{The Scientific World Journal}
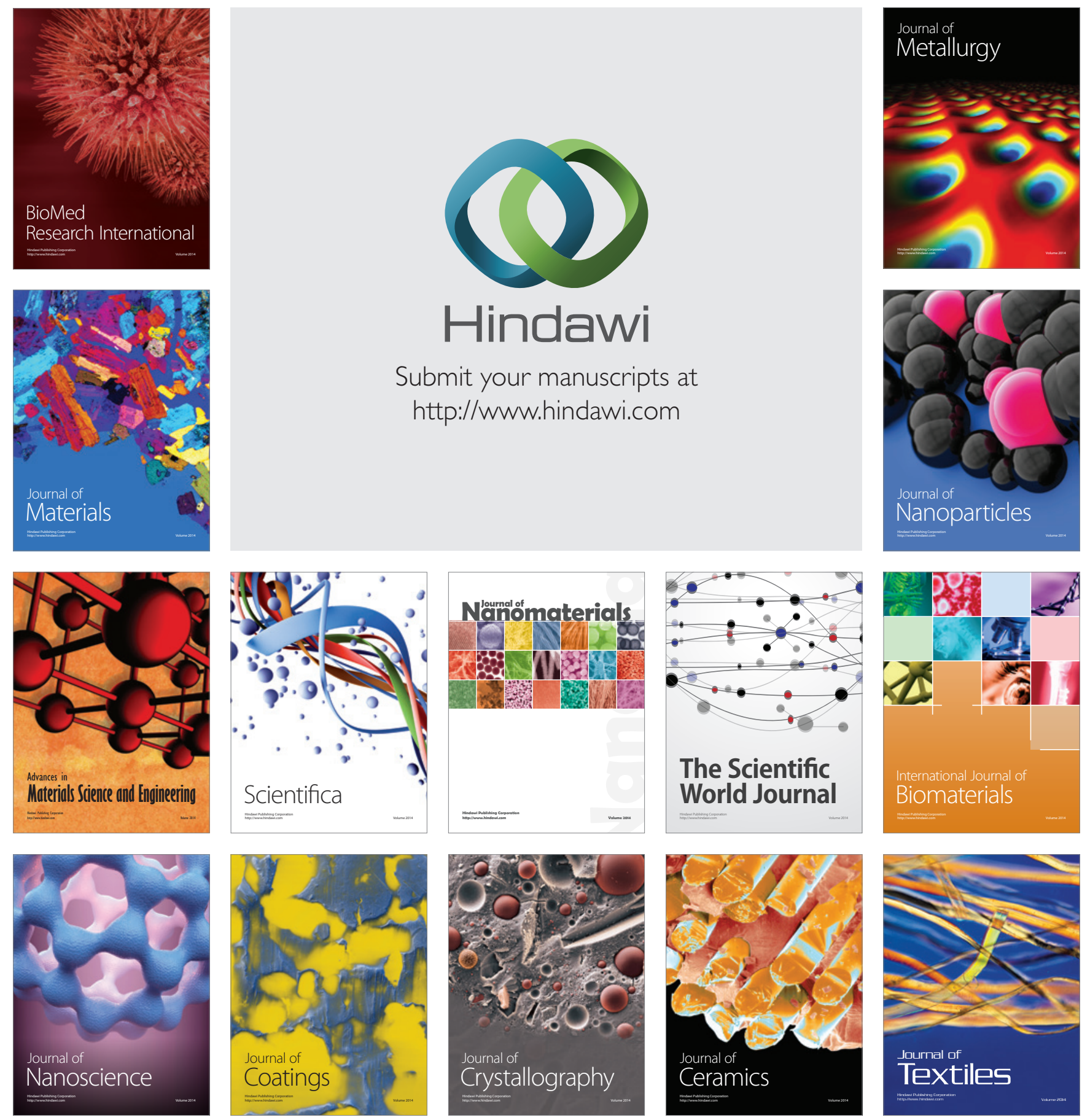